\title{
MEMBERSHIP LIST TO DATE
}

Abyberg, Walter (Ac.) Canadian International Paper

Ach, Edward K. (Ac.)

Co., 1155 Beaver Hall Square, Montreal, Que.

Canadian International Paper

Co., Forest Engineering Dept.

Victoria Bldg.,

Ottawa, Ont.

Adamson, M. A. (As.)

Addison, Peter (As.)

Alexander, Prof. J. L. (As.)

Alexander, John Playfair (As.)

Andrews, L. R. (Ac.)

Archer, C. F. (As.)

Ardenne, M. (As.)

Atkinson, C. C. (Ac.)

Atkinson, W. F. V. (Ac.)

431 Broadview Ave.,

Midhurst, Ont.

Forestry Department, University of Washington,

Seattle, Wash.

Dominion Forest Service, Pincher Creek, Alta.

Auden, A. J. (As.) Fort William Paper Co.,

5903 Larch St.,

B. C. Forest Branch,

Vancouver, B.C.

Ontario Forestry Branch, Tweed, Ont.

Woods Dept., Abitibi Power \& Paper Co.,

Iroquois Falls, Ont.

404 Laurier Ave. E., Ottawa, Ont.

Avery, B. F. (Ac.) Union Bank Bldg.,

Fort William, Ont.

Abitibi Power \& Paper Co., Toronto, Ont. Box 308,

Ayers, B. K. (As. N.) 35 Auburn St., Concord, N.H.

Barkhouse, F. A. (As.) Asst. Supt. of Forests, N.B. Rly. Co., 223 Aberdeen St., Frederickton, N.B.

Barnes, Geo. H. (As.) Forest Branch,

Barr, Dr. P. M. (Ac.)

TBassett, E. W. (As.)

OBastín, L. L. G. (As.)

Batt, C. A. (Ac.)

Baxter, H. H. (As.)

Bayly, G. (As.)

Ontario Forestry Branch,

Bedell, Geo. H. D. (As.) Dominion Forest Service,

Beede, V. A. (Ac.) Brown Corporation,

Bentley, A. W. (Ac. N.) New Power \& Paper Utilities

$$
\text { Corp., }
$$

Best, Albert Leslie (As.)

Black, Robson (As.)

Blair, R. J. (As.)

Blefgen, T. F. (As.)

Blyth, Gerald G. (As.)

Boisvert, Geo. (Ac.)

Bonney, P. S. (Ac.) c/o Forest Service,

o District Forester,

Engineering Dept., Inter.

national Paper Co., Box 420, Campbellton, N.B.

Canadian International Paper

$$
\text { Co., }
$$

Victoria, B.C.

Victoria, B.C

Vancouver, B.C.

Noranda, Que.

Vancouver, B.C.

Toronto, Ont.

Quebec, Que.

Dauphin, Man.

Riviere du Loup, Que.

Dominion Forest Service, Canadian Forestry Association,

19 Customs Bldg.,

22 Hill St.,

Ontario Paper Company, 71 St. Peter St.,

Cornerbrook, Nfld.

Prairie River, Sask.

Ottawa, Ont.

Ormstown, Que.

Calgary, Alta.

Ottawa, Ont.

Quebec, Que.

Woods Dept., Pacific Mills, Ocean Falls, B.C. 
Boultbee, Richard (As.)

Bourget, M. Albert (Ac.)

Boutin, Fernand (Ac.)

Brodie, J. A. (Ac.)

Brown, L. L. (As.)

Brown, R. M. (Ac.)

Brule, Joseph-Donat (Ac.)

Bubie, W. L. (Ac.)

Buenger, C. C. (As.N.R.)

Burchett, E. P. (As.)

Burk, A. H. (As.)

Burrows, T. C. (As.)

Burrows, Theodore A. (Ac.)

Byers, Wm. (As.)

Cain, M. V. (As.)

Cameron, D. R. (Ac.)

Campbell, F. J. (As.)

Campbell, R. L. (Ac.)

Candy, R. H. (Ac.)

Carlisle, K. W. (As.)

Carman, R. S. (Ac.)

on Carson, Wm. Jas. (As.)

Cass, N. D. (Ac.)

चै वaulkins, John George

\& (As.)

Caverhill, P. Z. (Ac.)

Cermak, Frank (As.)

:

DChristie, C. R. (Ac.)

JChristie, Prof. H. R. (Ac.)

'Claridge, B. E. (Ac.)

Clark, Dr. J. F. (Ac. N.)

Clark, S. H. (As.)

Clarke, T. A. (Ac.)

Clutterbuck, Sir Peter (H.)

Coleman, J. R. B. (As.)

Commins, M. J. (Ac.)

Connell, A. B. (Ac.)

Coolidge, P. T. (N.R.) (As.)

Cooper, S. N. (Ac.)

Cosens, G. G. (Ac.)

Courtnage, R. A. (Ac.)
Ontario Forestry Branch,

86 Mountain Hill,

Room 257, Government Bldgs.,

Ontario Forestry Branch,

712 Metropolitan Bldg.,

Forest products Laboratories, University of B. C.,

Parry Sound, Ont.

Quebec, Que.

Quebec, Que.

Toronto 5, Ont.

Vancouver, B.C.

Vancouver, B.C.

St. Lawrence F. P. Association, Val Brillant, Que.

Wayagamack Pulp \& Paper Co., Three Rivers, Que.

Sheboygan, Wis., U.S.

c/o McCoy Wilson Ltd., Reid

Bay,

Abitibi Power \& Paper Co.,

Dominion Forest Service,

403 Scott Block,

3246 Eleventh Ave. W.,

Topaz Harbour, B.C.

Sudbury, Ont.

Entrance, Alta.

Winnipeg, Man.

Vancouver, B.C.

Price Bros. 8 Co.,

Dominion Forestry Branch,

55 Westmount Blvd.,

Canadian International Paper

Co., 1155 Beaver Hall Square,

Dominion Forestry Service,

Camp 10, V. L. M. \& Co.,

71 St. Francois Xavier St., Apt. No. 5 ,

Dominion Forest Service,

c/o Tourville Lbr. Co., Trinity

Bay, via Rimouski Wharf,

B. C. Forest Branch, 1267 Pendrell St.,

Canada Power \& Paper Co.,

Dominion Forestry Service,

2575 West 35th St.,

Hammermill Paper Co.,

976 West 6th St.

B. C. Forest Branch,

Chicoutimi, W. Que.

Ottawa, Ont.

Montreal, Que.

Montreal, Que.

Ottawa, Ont.

Cowichan Lake, B.C.

Iroquois, Ont.

Three Rivers, Que.

Ottawa, Ont.

Rimouski, Que.

Victoria, B.C.

Vancouver, B.C.

Grand Mere, Que.

Big River, Sask.

Vancouver, B.C.

Matane, Que.

Riverside, Cal., U.S.A.

Entrance, Alta.

Nelson, B.C.

Simla and Delhi, India

North Bay, Ont.

Toronto 8, Ont.

Sioux Lookout, Ont.

2392 Queen St. E.,

Bangor, Maine.

31 Central St.,

Pine Falls, Man.

Manitoba Paper Co.,

Spruce Falls Power \& Paper Co., Kapuskasing, Ont.

Canadian International Paper Co., Three Rivers, Que. 
Flieger, Prof. B. W. (Ac.) Dept. of Forestry, University of N.B.,

B. C. Forest Branch, Forest Engineering Dept., Canaadian International Paper Co., Oritario Forestry Branch, Canada Power 8 Paper Co., Woodlands Dept., Price Bros. \& Co.,

Canadian International Paper Co., 140 Wellington St.,

St. Lawrence Paper Mills,

513 Pacific Bldg.,

Gardiner, Harold (As.)

Garman, Eric Heaton (As.)

Gaylord, F. A. (Ac.) సָ Gibson, Prof. J. M. (Ac. Gill, C. B. (Ac.) Gilmour, J. D. (Ac.) Gimby, W. E. (As.) Goodfellow, A. W. (Ac.) Gormley, M. W. (As.) o

Gosselin, C. (Ac.)

Graham, Angus (Ac.) Tे

Otraham, A. H. (As.) Grainger, M. A. (Ac.) Ereeley, Col. W. B. (H.) छี

Greenwood, W. B. (Ac.)

Sregg, E. E. (As.)

$\approx$ Greggor, R. D. (As.)

Greig, Donald (Ac.)

Griffith, Braham G. (As.) Groenewold, Carl Albert (As.)

Groves, Thos. D. (Student Member)

Guerin, C.

B. (Ac.)

Gutches, G. A. (Ac.N.)

Haddow, W. R. (Ac.)

Hall, John R. H. (As.)
Forest Branch,

Woodlands Branch, A.P.W. Paper Co.,

Forestry Department, Univer. sity of N. B.,

Dominion Forest Service,

Anglo Canadian P. \& P. Co., 71 St. Peter St.,

Logging Division, Canada Power \&ै Paper Co.,

Box 593 , Forest Branch,

Quebec P. 8 P. Mills Ltd.,

Quebec Forest Industrial Assoc., Ltd., 65 St. Anne St.,

16 Fraser Bldg.,

Metropolitan Bldg.,

c/o West Coast Lunbermen's Assoc., 245 Henry Bldg.,

Ontario Forestry Branch,

B.C. Forest Branch,

B.C. Forest Branch,

Brown Corp., 71 St. Peter St.,

Forest Branch, Dept. of Lands,

St. Lawrence Paper Mills,

4574, 6th Ave. W.,

Southern St. Lawrence Forest

'Prot., Association, 126 St.,

Peter St.,

3916 Jenifer St.,

Arnold Arboretum,

Dominion Forest Service,
Forest Survey Office, B.C.
Fredericton, N.B.

Vancouver, B.C.

Three Rivers, Que.

Port Arthur, Ont.

Casey, Que.

Chicoutimi, Que.

Ottawa, Ont.

Three Rivers, Que.

Vancouver, B.C.

Victoria, B.C.

Sheet Harbor, N. S.

Fredericton, N.B.

Swan River, Man.

Quebec, Que.

Grand Mere, Que.

Campbellton, N.B.

Victoria, B.C.

Chicoutimi, Que.

Quebec, Que.

Ottawa, Ont.

Vancouver, B.C.

Seattle, Wash.

North Bay, Ont.

Prince Rupert, B.C.

Victoria, B.C.

Quebec, Que.

Victoria, B.C.

Three Rivers, Que.

Vancouver, B.C.

Quebec, Que.

Washington, D.C.

Jamaica Plain, Mass.

Slave Lake, Alta. 
Halliday, W. E. D. (Ac.) Dominion Forestry Branch,

Hansson, Uno (As.)

Harris, Austin S. (As.)

Harrison, F. A. (As.)

Harrison, J. D. B. (Ac.)

Hart, H. V. (Ac.)

Heaney, Harold D. (As.)

Heimburger, C. C. (Ac.)

Hodgins, Hugh J. (As.)

Holloway, E. S. (As.)

Holman, H. L. (Ac.)

Hooper, W. A. (As.)

Horton, F. H. (As.)

Hosie, R. C. (Ac.)

Howe, Dr. C. D. (Ac.)

Howie, Eugene L. (As.)

Hudon, J. Eric (As.)

Huestis, Eric S. (As.)

Hughson, H. M. (Ac.)

Hume, John Fred, Jr.

\section{(Student Member)}

政Hutchison, J. A. (As.)

Irwin, C. H. (Ac.)

Irwin, J. C. W. (As.)

Jackson, F. H. R. (As.)

Jacombe, F. W. H. (Ac.)

Jago, R. D. (Ac.)

Jamer, D. W. (As.)

Jenkins, F. T. (Ac.)

Jenkins, J. H. (Ac.)

Jewett, C. A. (Ac.)

Johnson, J. W. (As.)

Johnston, R. N. (Ac.)

Johnson, Prof. Tor. (H.) Greenbush

c/o S. C. Hayden, 521 Winch Bldg.,

16 Fraser Bldg.,

Canadian International Paper Co., 140 Wellington St.,

Dominion Forest Service, Customs Bldg.,

St. Regis Paper Co., 1009 Themis Bldg.,

Canada Power \& Paper Co.

Cornell University, Dept. of Agriculture,

B.C. Forest Service, Parliament Bldgs.,

Dominion Forest Service,

Ontario Forestry Branch,

68 York St.,

Faculty of Forestry, Univer. sity of Toronto,

Faculty of Forestry, University of Toronto,

International Paper Co.,

$181 / 2$ Langevin St.,

'Dominion Forest Service,

Ottawa Electric Bldg.,

School of Forestry, University of Idaho,

Dominion Forest Service, 17 Customs Bldg.,

via Prairie River, Sask.

Vancouver, B.C.

Ottawa, Ont.

Ottawa, Ont.

Winnipeg, Man.

Montreal, Que.

Grand Mere, Que.

Ithaca, N.Y.

Victoria, B.C.

St. Hilaire, Que.

Calgary, Alta.

Kenora, Ont.

St. Catharines, Ont.

Toronto 5, Ont.

Toronto 5, Ont.

Campbellton, N.B.

Hull, Que.

Pincher Creek, Alta.

Ottawa, Ont.

Moscow, Idaho.

Calgary, Alta.

Laurentian Forest Protective As., 126 St. Peter St:

33 Glenwood Ave.,

Quebec, Que.

Toronto, Ont.

Dominion Forest Service,

Dominion Forest Service,

Price Bros. Eै Co.,

'c/o James McLaren Co.

Newfoundland Power \&8 Paper

Utilities Corporation

Forest Products Laboratory, University of B.C.,

Dominion Forest Service

'c/o Research Division, Forest Service, Dept. of the Interior

Ontario Forestry Branch

Entrance, Alta.

Ottawa, Ont.

Chicoutimi W., Que.

Buckingham, Que.

Cornerbrook, Nfld.

Vancouver, B.C.

Ottawa, Ont.

Ottawa, Ont.

Toronto 5, Ont.

Djursholm, Sweden. 
Kay, James (As.)

Kelleher, Geo. Francis (Ac.)

Kennedy, Howard (As.)

Kensit, N. M. (As.)

Kiefer, Francis (Ac.)

Kieffer, Henri (Ac.)

Kilby, W. H. (As.)

King, C. K. (As.)

Kinghorn, H. C. (Ac.)

Kingston, G. A. (As.)

Kishbaugh, Walter (Ac.)

Knapp, Prof. F. M. (Ac.)

Ḱoroleff, A. M. (Ac.)

Kynoch, W. (Ac.N.)

Lane, G. R. (Ac.)

Lane-Poole, C. E. (H.)

Langballe, N. B. (Ac.)

Lauderburn, D. E. (N.R.) (Ac.)

Leavitt, Clyde (Ac.)

Legat, C. E. (H.)

Le May, P.V. (As.)

Lessard, Eugene (Ac.)

Lewis, R. G. (Ac.)

Linekin, M. G. (Ass.)

Linton, G. M. (Ac.)

Lister, L. S. (As.)

Lockhart; George Arthur (As.)

Loomis, R. D. (Student)

Lovat, Major Gen. Lord (H)

Lussier, Omer (Ac.)

Lyford, C. A. (N.Ac.)

Lyford, P. L. (Ac.)

Lynum, Harold (Ac.)

Lyons, R. W. (Ac.)

McCallum, A. W. (Ac.)
General Delivery,

Box 252

c/o E. B. Eddy Co. Ltd.

Ontario Forestry Branch

14 Prince Arthur Ave.

Lands $छ$ Forests Dept.

Chief of Forest Protection, C.N.R.

N.B. International Paper Co.

Box 201

$1121 / 2$ Lisgar St.

Abitibi Power \& Paper Co.

Dept. of Forestry, University of B.C.

Canadian Pulp \& Paper Association, 701 Drummond Bldg.

School of Forestry,

University of Michigan, Ann Arbor, Mich.

Canadian Power \& Paper Co. Grand Mere, Que.

Commonwealth Forestry Advisor Canberra, Australia.

Howard Smith Paper Mills Oskelaneo, Que.

208 Old Capitol,

Board of Rly. Commissioners,

Chief Conservator of Forests, Union Bldg.

156 Percy St.

Dominion Bureau of Statistics, Ottawa, Ont.

Canadian International Paper Co. Three Rivers, Que.

Ontario Forestry Branch, Orono, Ont.

Hammermill Paper Co., Matane, Que.

Customs Bldg.

Dominion Forest Service, Winnipeg, Man.

Forestry Dept.,

University of N.B.

Under Secretary of State for Dominions,

Fredericton, N.B.

London, England.

Giffard, Que.

Jas. D. Lacey Co., Henry Bldg., Seattle, Wash.

Clark \& Lyford, Vancouver Blk. Vancouver, B.C.

1461 Burnaby St.,

Vancouver, B.C.

Spruce Falls Power \& Paper Co. Kapuskasing, Ont.

Central Experimental Farm, Ottawa, Ont. 
Morison, Murray B. (As.) Dominion Forest Service Morse, C. H. (Ac.) Dominion Forest Service Mulholland, F. D. (Ac.) B.C. Forest Branch Mulloy, G. A. (Ac.) Dominion Forest Service Munro, Donald J. (Ac.) c/o St. Anne Power Co.,

Newman, F. S. (Ac.) Ontario Forestry Branch Nisbet, R. H. (Ac.) Price Bros. \& Co.

Orchard, C. D. (Ac.) B.C. Forest Branch O'Connor, P. A. (As.)

Parker, H. A. (Ac.) Parlow, A. E. (Ac.) Parsons, H. H. (As.) Pepler, W. A. E. (Ac.)

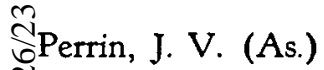
广Perry, R. S. (As.) б ○Phipps, Geo. W. (Ac.) WPiche, G. C. (Ac.) Pickard, T. D. (As.) aे opickford, A. E. (As.) Pike, R. T. (As.) T-Blahte, Frithjof Fonce, G. H. (Ac.) BRrowd, E. B. (Ac.) Fulling, A. V. S. (N.Ac.) 西位nam, M. M. (As.) 品

ERzacine, Eudore (Ac.) ORegan, Chas. E. (As.) Rensta, Gustav (Ac.) Reynolds, Henry George Edmund (As.)

Richards, E. S. (As.) Richardson, A. H. (Ac.) ERiley, Clifford George (As.)

Roberts, E. H. (Ac.)

ठำ I Ross, N. M. (Ac.)
Dominion Forest Service

Dominion Forestry Branch

E.C. Forest Service

246 Windermere Ave.

140 Wellington St. c/o International Paper Co.

Brown Corporation 71 St. Peter St.

Forest Products Laboratories University of B.C.

clo Spruce Falls Power \& Paper Co.

Quebec Forest Service

clo Canada Power 8 Paper Corp., Victoria Bldg.

B.C. Forest Branch Dominion Forestry Branch

Tostrups gate 22

Dept. of Lands and Mines

B.C. Forest Branch

1701 Regent St.

Canadian International Paper Co., 140 Wellington St.

Quebec Forest Service

91 First Ave. E.

607 Castle Bldg.

B.C. Forest Branch

Mersey Paper Co., Dennis Bldg.

Ontario Forestry Branch

Research Division, B. C. Forest Branch

Dominion Forest Service Box 812

Dept. of the Interior Forest Service

Dominion Forestry Branch
Marchand, Man.

Calgary, Alta.

Victoria, B.C.

Ottawa, Ont.

Beaupre, Que.

St. Williams, Ont.

Quebec, Que.

Victoria, B.C.

Winnipeg, Man.

Maple Creek, Sask.

Prince Rupert, B.C.

West Toronto, Ont.

Ottawa, Ont.

Quebec, Que.

Vancouver, B.C.

Kapuskasing, Ont.

Quebec, Que.

Ottawa, Ont.

Victoria, B.C.

Prince Albert, Sask.

Oslo, Norway

Fredericton, N.B.

Victoria, B.C.

Madison, Wis.

Ottawa, Ont.

Chicoutimi, Que.

North Bay, Ont.

Montreal, Que.

Nakusp, B.C.

Halifax, N.S.

Toronto, Ont.

Victoria, B.C.

Prince Albert, Sask.

Ottawa, Ont.

Indian Head, Sask. 
Rothery, J. E. (As. N.R.) International Paper Co.

Rowe, C. A. (As.)

Roy, Henri (Ac.)

Roy, J. A. (Ac.)

Ryan, Thomas Harold (As.)

Sabbaton, F. A. (As.)

Sanford, A. R. (As.)

Saunders, E. G. (Ac.)

Savage, H. B. (Ac.)

Scandrett, W. L. (As.)

Schanche, H. G. (Ac.)

Schierbeck, Otto (Ac.)

Schenstrom, Stig R: son (As.)

Scheult, Louis R. (As.)

Schubert, B. L. (Ac. N.R.)

Seaborne, R. L. (As.)

Seely, H. E. (Ac.)

Sharpe, J. F. (Ac.)

Shaw, Earle E. (Ac.)

ن.

Simmons, J. F. L. (As.)

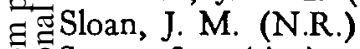

Oonart, Jas. (Ac.)

¿ Smith, Alfred G. (As.)

Of Smith, E. E. (Ac.)

Smith, Kaare (Ac.)

Soderston, Herbert R. (Ac.)

Sorgius, Henry (As.)

Sparks, N. C. (As.)

Springer, J. B. (As.)

Steele, W. E. (As.)

Stevenson, Col. H. I. (Ac.)

Stewart, J. V. (As.)

Stewart, K. A. (Ac.)

Stuart, R. V. (Ac.)

\section{East 42nd St.}

Canadian International Paper Co.

28 Kitchener St.

57 Scott St.

Box 340

Dominion Forest Service,

Canada Power \& Paper Co.

Canadian International Paper Co., 140 Wellington St.

Dominion Forest Service

Forest Engineers' Dept.

Canadian International Paper Co.

Box 242

Abitibi Power E Paper Co. Box 308

Provincial Forester

B.C. Forest Branch, Research Dept.

c/o Canadian Power \& Paper Corp., 140 Wellington St.

377 Pleasant St.

Mersey Paper Co. Ltd.

c/o R. E. Rodney, 227 Nepean St.

Ontario Forestry Branch

Canadian International Paper Co.

Ontario Forestry Branch

431 West Broad St.

Dominion Parks Branch

Box 57, Rocky Mountain House, Alta.

International Power \& Paper Co. Cornerbrook, Nfld.

Pejepscot Paper Co.

Abitibi Power $E^{2}$ Paper Co.

86 University Ave.

45 Maple Ave.

Dominion Parks Branch

c/o Jas. McLaren Co.

Logging Division, Canadian Pulp $\&$ Paper Co.,

Dominion Forest Service

Ontario Forestry Branch

Ontario Forestry Branch

921 Metropolitan Bldg.
New York

St. Jovite Sta., Que.

Quebec, Que.

Quebec, Que.

Kamloops, B.C.

Grand Mere, Que.

Ottawa, Ont.

N. Westminster, B.C.

Three Rivers, Que.

London, Ont.

Toronto, Ont.

Halifax, N.S.

Victoria, B.C.

Ottawa, Ont.

Brockton, Mass.

Liverpool, N.S.

Ottawa, Ont.

Toronto, Ont.

Gaspe Harbour, Que.

Toronto, Ont.

Hazleton, $\mathrm{Pa}$.

Dauphin, Man.

Cookshire, Que.

Toronto

Shawinigan Falls, Que.

Ottawa, Ont.

Buckingham, Que.

Grand Mere, Que.

Winnipeg, Man.

Fort Frances, Ont.

Sudbury, Ont.

Vancouver, B.C. 
Sutherland, J. W. (As.)

Swaine, Dr. J. M. (As.)

St. Clair, R. C. (Ac.)

Teasdale, John A. (As.) 607 Castle Bldg.

Tessier, Gustave A. (Ac.) 105 Murray Ave.

Thomson, G. J. (As.)

Thrupp, A. C. (Ac.)

Tilt, L. C. (Ac.)

Townsend, C. R. (Ac.)

Tremblay, T. A. V. (As.)

Tucker, J. E. (As.)

Tunstell, George (Ac.)

Turnbull, J. F. (Ac.)

Turner, Jack (N.As.)

Unwin, Dr. A. H. (N.Ac.)

Van Camp, J. L. (Ac.)

Van Dusen, W. J. (Ac.)

Veness, J. C. (Ac.)

ล

Walton, John R. (Ac.)

Watt, R. M. (Ac.)

WWebb, H. P. (Ac.)

JWWebb, L. S. (Ac.)

\$W Westland, C. E. (Ac.)

White, C. E. (As.)

White, Dr. J. H. (Ac.)

WWhitelaw, W. A. (As.)

Wightman, A. F. (As.)

Wilkinson, T. H. (As.)

Wilson, Ellwood (Ac.)

Wilson, F. G. (N.Ac.)

Wilson, Jas. Clarence

(As.)

Wilson, J. O. (Ac.)

Wilcox, F. R. (Ac.)

Willson, W. E. (As.)

Co.

Box. 37

73 Jameson Ave. tion

Price Bros.

Box 420

Box 357
Canadian International Paper

Entomological Branch

B.C. Forest Branch,

Forestry Dept. Can. Power \&?

Paper Co., Wayagmack Island. Three Rivers, Que.

Howard Smith Paper Mills Sudbury, Ont.

Dominion Forestry Branch Winnipeg, Man.

clo International Lumber Co. Campbell River, B.C.

Orillia, Ont., R.R.

Anglo Newfoundland Dev. Co. Grand Falls, Nfld.

Principal Forest Officer

Nicosia, Cyprus, Via Port Said

Dominion Forest Nursery Sta-

712 Metropolitan Bldg.

Dominion Forest Service

Dominion Forestry Branch

Canada Power \& ${ }^{3}$ Paper Co.

Ontario Forestry Branch

Dominion Forest Service

Faculty of Forestry University of Toronto

International Paper Co. 140 Wellington St.

clo Rev. F. A. Wightman

B.C. Lumber Manufacturers Assoc., Metropolitan Bldg.

Canada Power \& ${ }^{3}$ Paper Corp.

clo Canada Power \& Paper Corp., 601 Victoria Bldg. 140 Wellington St.

43 Moncton Ave.

Room 707, Victoria Bldg., 140 Wellington St.

Woods Dept., Abitibi Power \& Paper Co.
Indian Head, Sask.

Vancouver, B.C.

Ottawa, Ont.

Chicoutimi, Que.

Dauphin, Man.

Grand Mere, Que.

Campbellton, N.B.

Toronto, Ont.

Coalspur, Alta.

Toronto, Ont.

Ottawa, Ont.

Milltown, N.B.

Vancouver, B.C.

Grand Mere, Que.

Madison, Wis.

Ottawa, Ont.

Ottawa, Ont.

Iroquois Falls, Ont.
Quebec, Que. 
Winegar, B. M. (Ac.) Canada Creosoting Co.,

Wright, C. C. (Student) Canada Cement Bldg.

345 Waverley St.

Price Bros. \& Co.

Montreal, Que.

Ottawa, Ont.

Zavitz, E. J. (Ac.) Ontario Forestry Branch Toronto, Ont.

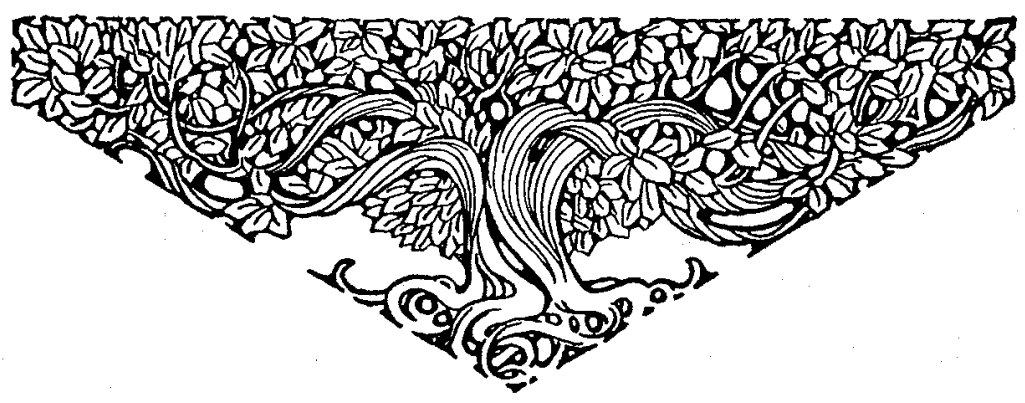

